\title{
Gas-phase ion chemistry in the 21st century
}

\author{
Fulvio Cacace \\ Dipartimento di Studi di Chimica e Tecnologia delle Sostanze Biologicamente Attive, Università \\ di Roma "La Sapienza", P. le A. Moro, 5 - 00185 Rome, Italy
}

\begin{abstract}
Biography: Fulvio Cacace was born in Rome in 1931 and received his undergraduate education at the University of Rome, as a student of Prof. G. Giacomello. Then followed postdoctoral experience at Uppsala, Brookhaven National Laboratory, and Kernforschungauslage Jülich and teaching appointments at the Universities of Camerino and Viterbo. Since 1971 he has been Professor of General Chemistry at the University of Rome. His principal research interests include kinetic and thermochemical aspects of gas-phase ion chemistry.
\end{abstract}

I intend to discuss here the foreseeable contribution of gas-phase ion chemistry to the development of physical organic chemistry in the 21 st century. Whereas such a choice undoubtedly reflects my own research interests, it is amply justified by the innovative character of the conceptual and experimental tools provided by gas-phase ion chemistry, and by the potential scope of its applications. Perhaps the aspect most widely appreciated at the present time by physical organic chemists is the thermochemical study of gaseous ions, which has helped redress certain trends distorted in solution by differential-solvation effects and has provided generalized acidity and basicity scales unperturbed by the effects of the environment. This line of research will certainly continue to flourish in the near future, owing in particular to the fact that only gasphase experiments provide structural, thermochemical, and reactivity data directly comparable to those from theoretical methods (1).

Gas-phase ionic kinetics are less familiar to the physical organic chemists, a situation that is likely to change in the 21 st century in view of the rapidly expanding role of gas-phase studies. At the low-pressure extreme, investigated with experimental techniques such as ion-cyclotron resonance mass spectrometry, ionic reactions are dominated by long-range electrostatic forces and occur under isolated-pair conditions. Their kinetic treatment has required the development of specialized models (2), and has promoted the analysis of the processes, e.g., radiative cooling (3), that allow stabilization of excited ions in the absence of thermalizing collisions. At the high-pressure extreme, up to now accessible only to techniques other than mass spectrometry such as $\beta$-decay and radiolytic methods whose range extends well above atmospheric pressure (4), ionic reactions obey instead standard thermal kinetics, and the study of important classes of reactions has revealed patterns more familiar to physical organic chemists. For example, it has been found that gas-phase aromatic substitution displays orientation and selectivity trends fully comparable to those observed in solution, and it has been possible to measure the activation parameters of many thermal ionic processes, including proton and hydride-ion transfer, carbenium ions isomerization, etc. In recent years, however, a significant change of emphasis has become noticeable in gas-phase studies, initially perceived as a long-awaited opportunity to get rid of the solvent, regarded as an adventitious factor that affects the intrinsic reactivity pattern and hinders the theoretical analysis of the reaction. Rapidly accumulating evidence has shown instead that the presence of the solvent, even if limited to a single molecule, is often necessary for the reaction to occur, at least in the way long familiar to physical organic chemists; e.g., bare $\mathrm{NO}_{2}^{+}$undergoes in the gas-phase only charge and oxygen-atom transfer to arenes, whereas in the presence of a single molecule of $\mathrm{H}_{2} \mathrm{O}$ or $\mathrm{CH}_{3} \mathrm{OH}$ it is a wellbehaved nitrating agent (5). Accordingly, increasing efforts are currently being devoted to the study of ionic reactions, not in the absence of the solvent but in a "microsolvated" environment, namely in tailor-made charged complexes containing a well-defined number of neutral molecules. Many techniques have already been developed to this end. An ingenious approach is based on the interaction of an ion with a molecule characterized by the presence of two reactive centers connected by a flexible link. The one not directly involved in the reaction and denoted as "spectator" effectively solvates the reacting center (6). Another approach involves addition of an ion to a neutral molecule in a gas at sufficiently high pressure (760 torr) to 
cool the adduct and prevent its dissociation, so that the reaction occurs within a long-lived complex. Other effective techniques are low-temperature chemical ionization and flowing-afterglow mass spectrometry, operating in a pressure range up to several torr, which allow formation and mass selection of ionic clusters of variable size, and the study of intracluster reactions. A most interesting alternative is to produce neutral van der Waals clusters by supersonic expansion, and then to ionize one of their molecules by electron impact or multiphoton absorption and study the ensuing intracluster reactions by optical or mass spectrometry (7). The development of these techniques has opened a new research field that represents a kind of gas-phase supramolecular chemistry and can be most useful as an innovative approach to the chemistry of solvation, a longstanding issue in physical organic chemistry. In particular, gaseous clusters are the most suitable medium for understanding the details of solvent-reactant interactions which are lost in bulk-phase studies, owing to the averaging of configurations and energies typical of large-scale molecular ensembles. The first results, which pertain exactly to the area traditionally covered by physical organic chemistry, are undoubtedly encouraging. Among many relevant examples one can cite the "Crafts-Friedel" reaction, namely the electrophilic alkylation process taking place in long-lived ion-molecule complexes formed upon addition of arenium ions to olefins, whose occurrence had long been predicted, but which has been demonstrated only very recently by exploiting the special features of the gaseous reaction environment (8). Other significant examples are the ion-molecule reactions within mixed $\left[\left(\mathrm{H}_{2} \mathrm{O}\right)_{\mathrm{n}} \mathrm{HNO}_{3} \mathrm{NO}_{2}^{+}\right]$clusters, of great relevance to atmospheric chemistry (9), and the $\mathrm{S}_{\mathrm{N}} 2$ reactions within ionized halobenzene-ammonia and halobenzene-methanol clusters (7). Another application of considerable interest to the problem of enantiomeric recognition and differentiation is related to ionic reactions in gaseous clusters formed by chiral molecules, currently studied by laser-induced fluorescence-excitation techniques (10). There is little doubt that ionic gas-phase supramolecular chemistry will develop in the 21 st century into a major research area, and will significantly contribute to disentangle the intrinsic and the environmental factors whose combination governs the chemical reactivity.

Having outlined several of the most promising themes and methodologies of gas-phase ion chemistry, I would like to mention a few significant areas where, in my opinion, it may open new perspectives to the physical organic chemistry of the 21 st century. To the colleagues worried about the reputedly shrinking area of application of their discipline, I would recommend reading the recent book entitled The Chemically Controlled Cosmos, which reviews the current status of astrochemistry, emphasizing the central role, not generally appreciated in all of its far-reaching aspects, of interstellar molecules and ions (11). These species, mostly organic, discovered in a large and rapidly increasing number by optical and radio spectroscopy, are not only most relevant to the problem of the origin and dissemination of life in the Universe but, perhaps more importantly, are a key factor that controls the evolution of astrophysical objects, in particular the birth of stars from interstellar clouds. The radiations emitted by the molecules cool the cloud, and set its temperature, which allows condensation to form the star. Furthermore, the ionization of a fraction of the molecules ensures the coupling of the cloud to magnetic and electric fields, which is crucial to control gravitational collapse, establishing the delicate equilibrium required for the birth of the star. The picture contains all the typical ingredients, namely molecules, ions (mostly organic), and their interactions, which make interstellar space the largest reaction "vessel" for gas-phase ion-molecule processes. The current status of astrochemistry and its foreseeable development promoted by the rapid accumulation of data from the new observational techniques pose a number of fundamental problems whose solution is necessary for the elaboration of astrophysical models as well as for the advancement of the research on the origin of life. Most of the problems, related to interstellar molecules and ions, particularly as concerns their formation processes, reactivity, absorption and emission of radiation, etc., pertain to physical organic chemistry and call for the application of the theoretical and experimental techniques of gas-phase ion chemistry.

Planetary chemistry is also a fascinating field where again physical organic chemistry is expected to play a central role. Consider, as an example, the atmosphere of Jupiter with its large content of methane, subjected to an intense flux of ionizing radiations; i.e., cosmic rays and short-wavelength photons. Such a gaseous environment, characterized by an extended pressure range and very cold and dense near the surface of the planet, is a huge natural reactor where carbenium-ion chemistry occurs on a large scale, producing organic cations in amounts that dwarf not only the minute quantities formed in the ion sources of the chemical ionization mass spectrometers or in NMR tubes containing superacid solutions, but, by orders of magnitude, the output of any man-made chemical plant. The kinetic and mechanistic study of this very special branch of carbenium-ion chemistry, performed in the laboratory under conditions approaching those of planetary atmospheres, is another significant research area for the physical organic chemistry of the 21 st century. Its relevance can be expected to increase as more chemical data will be provided by the unmanned planetary 
missions already scheduled for the next decade and, in turn, the results of the laboratory experiments will prove essential for planning the missions of manned space vehicles later in the 21 st century.

Down to the earth, there are several research fields open to the skills of physical organic chemists willing to exploit the resources already available in the armory of gas-phase chemistry. I'm thinking of atmospheric chemistry, combustion, flame, and plasma chemistry, the latter one particularly promising in connection with recent advances in innovative-materials research.

I conclude this contribution with the cautionary note that many of the areas cited are outside the classic domain of physical organic chemistry, and their study calls for unusual and innovative approaches. Nevertheless, I perceive physical organic chemistry not as a circumscribed branch of chemistry but as a general strategy for the solution of chemical problems by physical methods and tools. As such, it has repeatedly demonstrated its ability to adjust to changing times, and I don't see any reasons why it should fail to display the same flexibility in facing the problems, however demanding, of the 21 st century.

\section{REFERENCES}

1. B. J. Smith and L. Radom. J. Am. Chem. Soc. 115, 4885 (1993).

2. J. I. Brauman, W. N. Olmstead and C. A. Lieder. J. Am. Chem. Soc. 96, 4030 (1974).

3. Y.-W. Cheng and R. C. Dunbar. J. Phys. Chem. 99, 10802 (1995).

4. J. M. Farrar and W. H. Saunders, Eds. Techniques for the Study of lon-Molecule Reactions, Techniques of Chemistry Series, Vol. 20, Wiley, New York (1988).

5. M. Aschi, M. Attina, F. Cacace and A. Ricci. J. Am. Chem. Soc. 116, 9535 (1994), and references therein.

6. M. E. Crestoni, S. Fornarini and D. Kuck. J. Phys. Chem. 99, 3144 (1995).

7. B. Brutschy. Chem. Rev. 92, 1567 (1992).

8. M. Aschi, M. Attina and F. Cacace. J. Am. Chem. Soc. 117, 12832 (1995).

9. Y. Cao, J.-H. Choi, B.-M. Haas and M. Okumura. J. Phys. Chem. 98, 12176 (1994).

10. A. R. Al-Rabaa, E. Breheret, F. Lahmani and A. Zehnacker. Chem. Phys. Lett. 237, 480 (1995).

11. T. W. Hartquist and D. A. Williams, The Chemically Controlled Cosmos: Astronomical Molecules from the Big Bang to Exploding Stars, Cambridge University Press (1995). 